\title{
Symmetry Classification of Energy Bands in Graphene and Silicene
}

\author{
Eugene Kogan \\ Department of Physics, Bar-Ilan University, Ramat-Gan, Israel \\ Email: Eugene.Kogan@biu.ac.il
}

Received February 18, 2013; revised March 19, 2013; accepted April 13, 2013

Copyright (C) 2013 Eugene Kogan. This is an open access article distributed under the Creative Commons Attribution License, which permits unrestricted use, distribution, and reproduction in any medium, provided the original work is properly cited.

\begin{abstract}
We present the results of the symmetry classification of the electron energy bands in graphene and silicene using group theory algebra and the tight-binding approximation. The analysis is performed both in the absence and in the presence of the spin-orbit coupling. We also discuss the bands merging in the Brillouin zone symmetry points and the conditions for the latter to become Dirac points.
\end{abstract}

Keywords: Graphene; Silicene; Group Theory; Dirac Points

\section{Introduction}

Since graphene was first isolated experimentally [1], it is in the focus of attention of both theorists and experimenttalists. Obviously, understanding of the symmetries of the electrons dispersion law in graphene is of crucial importance. Actually, the symmetry classification of the energy bands in graphene (or "two-dimensional graphite") was presented nearly 60 years ago by Lomer in his seminal paper [2]. Later the subject was analyzed by Slonczewski and Weiss [3], Dresselhaus and Dresselhaus [4], Bassani and Parravicini [5]. Recent approaches to the problem are presented in the papers by Malard et al. [6], Manes [7] and in our publication [8].

The present work has two aspects: a pragmatic and a pedagogical one. The first aspect is connected with the recent synthesis of silicene, the counterpart of graphene for silicon, with buckled honeycomb geometry. This novel two-dimensional material has attracted recently considerable attention, both theoretically $[9,10]$ and experimentally, due to its exotic electronic structure and promising applications in nanoelectronics as well as its compatibility with current silicon-based electronic technology. So we present the symmetrty analysis of the silicene electron bands.

The pedagogical aspect is connected with the fact that different approaches to the symmetry classification, even if giving the same results, are based on different methods of applications of group theory. Thus in our previous paper [8] the labeling of the bands was based on com patibility relations and guesses. In the present work we show that in the framework of the tight-binding approximation the representations of the little group in the symmetry points can be rigorously found in the framework of the group theory algebra. Though the idea of using the tight-binding approximation is by no means new (it was used already in the work by Lomer), our mathematical approach is totally different, as one can easily see comparing the present work with [2], and, to our opinion, more convenient for applications. This statement is supported by the analysis of the symmetry of the energy band in silicene.

We also generalize the symmetry classification by taking into account the spin-orbit coupling both for graphene and for silicene. This, to the best of our knowledge, wasn't done before even for graphene. Though in graphene the spin-orbit coupling is very weak, the problem is interesting in principle. One can expect that in silicenr the coupling is stronger, and it will become even more so for graphene related materials from heavier elements, provided they can be synthesized.

To remind to a reader a few basic things, important for the symmetry classification of the bands in any crystal, consider a point sub-group $R$ of the space group characterizing the symmetry of a crystal (we restrict ourselves with the consideration of symmorphic space groups). Any operation of the group $R$ (save the unit transformation) takes a general wavevector $\boldsymbol{k}$ into a distinct one. However, for some special choices of $\boldsymbol{k}$ some of the operations of the group $R$ will take $\boldsymbol{k}$ into itself rather than into a distinct wavevector. These particular operations are called the 
group of $\boldsymbol{k}$; it is a subgroup of the group $R$. Points (lines) in the Brillouin zone for which the group of the wavevector contains elements other than the unit element are called symmetry points (lines). We may use a state (states) corresponding to such a special wavevector to generate a representation for the group of $\boldsymbol{k}[11,12]$. In this paper we consider crystals with the hexagonal Brillouin zone. In this case the symmetry points are $\Gamma$ - the center of the Brillouin zone, the points $K$ which are corners of the Brillouin zone and the points $M$ which are the centers of the edges of the Brillouin zone.

\section{Tight-Binding Model}

We'll deal with the materials with a basis of two atoms per unit cell, and we'll search for the solution of Schroedinger equation as a linear combination of the functions

$$
\psi_{\beta ; \boldsymbol{k}}^{j}=\sum_{R_{j}} \psi_{\beta}\left(\boldsymbol{r}-\boldsymbol{R}_{j}\right) \mathrm{e}^{\mathrm{i} \boldsymbol{k} \cdot \boldsymbol{R}_{j}},
$$

where $\psi_{\beta}$ are atomic orbitals, $j=A, B$ labels the sublattices, and $\boldsymbol{R}_{j}$ is the radius vector of an atom in the sublattice $j$.

A point symmetry transformation of the functions $\psi_{\beta ; \boldsymbol{k}}^{j}$ is a direct product of two transformations: the transformation of the sub-lattice functions $\varphi_{\boldsymbol{k}}^{A, B}$, where

$$
\phi_{\mathbf{k}}^{j}=\sum_{\boldsymbol{R}_{j}} \mathrm{e}^{\mathrm{i} \boldsymbol{k} \cdot \boldsymbol{R}_{j}},
$$

and the transformation of the orbitals $\psi_{\beta}$. Thus the representations realized by the functions (1) will be the direct product of two representations. Generally, these representations will be reducible. To decompose a reducible representation into the irreducible ones it is convenient to use equation

$$
a_{\alpha}=\frac{1}{g} \sum_{G} \chi(G) \chi_{\alpha}^{*}(G),
$$

which shows how many times a given irreducible representation $\alpha$ is contained in a reducible one [13]. Additional information about the representations can be obtained if we use projection operator [14]

$$
O_{\alpha}=\frac{n_{\alpha}}{g} \sum_{G} \chi_{\alpha}^{*}(G) P(G),
$$

where $n_{\alpha}$ is the dimensionality of the irreducible representation $\alpha$ and $P(G)$ is the operator corresponding to a transformation $G$. The operator projects a given function to the linear space of the representation $\alpha$. For a one dimensional representation the operator thus gives basis of the representation.

\section{Group Theory Analysis in the Tight-Binding Model without the Spin-Orbit coupling}

Our tight-binding model space includes four atomic or- bitals: $|s, p\rangle$. Notice that we assume only symmetry of the basis functions with respect to rotations and reflections; the question how these functions are connected with the atomic functions of the isolated carbon atom is irrelevant.

\subsection{Graphene}

The Hamiltonian of graphene being symmetric with respect to reflection in the graphene plane, the bands built from the $|z\rangle$ orbitals decouple from those built from the $|s, x, y\rangle$ orbitals. The former are odd with respect to reflection, the latter are even. In other words, the former form $\pi$ bands, and the latter form $\sigma$ bands.

The group of wave vector $\boldsymbol{k}$ at the point $\Gamma$ is $D_{6 h}$, at the point $K$ is $D_{3 h}$, at the lines $\Gamma-K$ is $C_{2 v}[8,15]$. The representations of the groups $D_{3 h}$ and $D_{6 h}$ can be obtained on the basis of identities

$$
D_{3 h}=D_{3} \times C_{s}, D_{6 h}=C_{6 v} \times C_{s} .
$$

the irreducible representations of the group $D_{3}$ are presented in the Table 1. Each representation of the group, say $A_{1}$, begets two representations of the group $D_{3 h}$ : $A_{1}^{\prime}$ and $A_{1}^{\prime \prime}$; prime means that the representation is even with respect to reflection $\sigma_{h}$, double prime means that it is odd.

The irreducible representations of the group $C_{6 v}$ are presented in the Table 2. Because the inversion transformation $I$ can be presented as

$$
I=C_{2} \sigma_{h}
$$

the representations of the group $D_{6 h}$ can be classified as symmetric $(\mathrm{g})$ or antisymmetric $(\mathrm{u})$ with respect to inversion. Thus each representation of the group $C_{6 \mathrm{v}}$, say $A_{1}$, begets two representations of the group $D_{6 h}$ : $A_{1 g}$ and $A_{1 u}$.

Notice that the orbitals $|s\rangle$ (or $|z\rangle$ ) realize $A_{1}$ representation both of the group $D_{3}$ and of the group $C_{6 v}$, hence the representations of the groups realized by the functions $\psi_{s, z ; \boldsymbol{k}}^{A, B}$ will be identical to those realized by the sub-lattice functions $\varphi_{\boldsymbol{k}}^{A, B}$.

Let us start from the symmetry analysis at the point $\Gamma$. Because the transformations $C_{2}, C_{6}, \sigma_{v}$ change sublattices, the characters corresponding to these transfor of the group $C_{6 v}$.

Table 1. Characters table for irreducible representations of $D_{3}$ point groups.

\begin{tabular}{cccc}
\hline$D_{3}$ & $E$ & $2 C_{3}$ & $3 U_{2}$ \\
\hline$A_{1}$ & 1 & 1 & 1 \\
$A_{2}$ & 1 & 1 & -1 \\
$E$ & 2 & -1 & 0 \\
\hline
\end{tabular}


Table 2. Characters table for irreducible representations of $C_{6 v}$ point group.

\begin{tabular}{ccccccc}
\hline $\mathrm{C}_{6 \mathrm{~V}}$ & $\mathrm{E}$ & $\mathrm{C}_{2}$ & $2 \mathrm{C}_{3}$ & $2 \mathrm{C}_{6}$ & $2 \sigma_{v}$ & $3 \sigma_{v}^{\prime}$ \\
\hline $\mathrm{A}_{1}$ & 1 & 1 & 1 & 1 & 1 & 1 \\
$\mathrm{~A}_{2}$ & 1 & 1 & 1 & 1 & -1 & -1 \\
$\mathrm{~B}_{2}$ & 1 & -1 & 1 & -1 & 1 & -1 \\
$\mathrm{~B}_{1}$ & 1 & -1 & 1 & -1 & -1 & 1 \\
$\mathrm{E}_{2}$ & 2 & 2 & -1 & -1 & 0 & 0 \\
$\mathrm{E}_{1}$ & 2 & -2 & -1 & 1 & 0 & 0 \\
\hline
\end{tabular}

Mations are equal to zero. The transformations

$E, C_{3}, \sigma_{v^{\prime}}$ leave the sub-lattices as they were. Hence from Table 2, we see that the functions $\phi_{0}^{A, B}$ realizes reducible representation

$$
R_{\Gamma}=A_{1}+B_{2}
$$

Taking into account the symmetry of the states relative to reflection in the plane of graphene, we obtain that at the point $\Gamma$ the functions $\psi_{2}$ (here and further on, when this is not supposed to lead to a misunderstanding, we'll suppres the index $\boldsymbol{k}$ in $\psi_{\beta ; \boldsymbol{k}}$ ) realize $A_{1 u}$ and $B_{2 g}$ representations of the group $D_{6 h}$, characterizing $\pi$ band; the functions $\psi_{s}$ realize $A_{1 g}$ and $B_{2 u}$ representations of the group $D_{6 h}$, characterizing $\sigma$ band.

Acting by projection operators $O_{A}$ and $O_{B}$ on a function $\psi^{j}$, we obtain that the irreducible representation $A_{1}$ is realized by symmetric combination of the $A$ and $B$ orbitals, and the irreducible representation $B_{2}$ by the antisymmetric combination. One can expect that the first case occurs in the hole band, and the second in the electron band.

The orbitals $|x, y\rangle$ realize representation $E_{1}$ of the group $C_{6 v}$ [13]. Hence, representation of the group realized by the functions $\psi_{x, y}^{A, B}$ can be decomposed as

$$
E_{1} \times R_{\Gamma}=E_{1}+E_{2} .
$$

Taking into account the symmetry of the states relative to reflection in the plane of graphene, we obtain that at the point $\Gamma$ the functions $\psi_{x, y}$ realize $E_{1 u}$ and $E_{2 g}$ representations of the group $D_{6 h}$, characterizing $\sigma$ bands.

To find wavefunctions, realizing each of the irreducible representations, we apply the projection operators and obtain

$$
O_{E_{1}} \psi_{ \pm}^{j} \square \psi_{ \pm}^{j}+\psi_{ \pm}^{\bar{j}}, O_{E_{2}} \psi_{ \pm}^{j} \square\left(\psi_{ \pm}^{j}-\psi_{ \pm}^{\bar{j}}\right),
$$

where $\bar{j}=B$ if $j=A$, and vice versa. Thus representation $E_{1}\left(E_{2}\right)$ is realized by symmetric (antisymmetric) combinations of $|x, y\rangle$ orbitals. One can expect that the first representation is realized at the hole band, and the second at the valence band.

Now let us perform the symmetry analysis at the point $K$. The representation of the group realized by the functions $\phi_{K}^{A, B}$ is determined by the transformation law of the exponentials $\mathrm{e}^{\mathrm{i} K \cdot \boldsymbol{R}_{j}}$ under the symmetry operations. Rotation of the radius vector by the angle $2 \pi / 3$ anticlockwise, is equivalent to rotation of the vector $\boldsymbol{K}$ in the opposite direction, that is to substitution of the three equivalent corners of the Brillouin zone:

$\boldsymbol{K} \rightarrow \boldsymbol{K}_{2} \rightarrow \boldsymbol{K}_{3} \rightarrow \boldsymbol{K}$, where

$\boldsymbol{K}=(2 \pi / 3 a, 2 \pi / 3 \sqrt{3} a), \quad \boldsymbol{K}_{2}=(0,-4 \pi / 3 \sqrt{3} a)$ and

$\boldsymbol{K}_{3}=(-2 \pi / 3 a, 2 \pi / 3 \sqrt{3} a)$. The rotation multiplies each basis vector by the factor $\mathrm{e}^{2 \pi / 3}$. Using Eqation (3), we obtain

$$
a_{E}=(1 / 3)\left(2-\mathrm{e}^{2 \pi i / 3}-\mathrm{e}^{-2 \pi \mathrm{i} / 3}\right)=1 .
$$

Hence, the functions $\phi_{K}^{A, B}$ realize irreducible representation $E$ of the group $D_{3}$.

Taking into account the symmetry of the states relative to reflection in the plane of graphene, we obtain representation $E^{\prime \prime}$ of the group $D_{3 h}$, realized by the $\psi_{z}$ functions (merging $\pi$ bands), and representation $E^{\prime}$, realized by the $\psi_{s}$ functions, characterizing $\sigma$ bands.

The orbitals $|x, y\rangle$ realize representation $E$ of the group $D_{3}$ [13]. Hence, representation of the group realized by the quartet of functions $\psi_{x, y}^{A, B}$ can be decomposed as

$$
E \times E=A_{1}+A_{2}+E .
$$

taking into account the symmetry of the states relative to reflection in the plane of graphene, we obtain representations $A_{1}^{\prime}, A_{2}^{\prime}$ and $E^{\prime}$ of the group $D_{3 h}$, realized by the $\psi_{x, y}$ functions, characterizing $\sigma$ bands.

Acting by projection operators, we obtain that the representation $A_{1}$ is realized by the vector space with the basis vector $\psi_{+}^{A}+\psi_{-}^{B}$, and the representation $A_{2}$ is realized by the vector space with the basis vector $\psi_{-}^{A}-\psi_{+}^{B}$. The vector spaces realizing representations $A_{1}$ and $A_{2}$ being found, the representation $E$ is obviously realized by the vector space spanned by the vectors $\psi_{+}^{A}-\psi_{-}^{B}, \psi_{-}^{A}+\psi_{+}^{B}$.

Because the irreducible representation $E^{\prime}$ is realized both by $\psi_{s}$ and $\psi_{x, y}$ functions, these representations should be considered together. According to Wigner theorem [16] we still have two $E^{\prime}$ representations, each of them being realized by two functions from a quartet $\psi_{s}^{A}, \psi_{s}^{B}, \psi_{+}^{A}-\psi_{-}^{B}, \psi_{+}^{A}-\psi_{-}^{B}$. Each $E^{\prime}$ representation characterizes two $\sigma$ bands, merging at the point $K$.

The symmetry of the electron bands at the points $\Gamma$ and $K$ being determined, the symmetry at the lines $\Gamma-K$ follows unequivocally from the compatibility relations, presented in Table $3[8,13]$. The table shows 
compatibility of the representations of the point group $C_{2 v}$, realized at the symmetry line $\Gamma-K$, with those realized at the symmetry points $\Gamma$ and $K$.

The results of this section are presented on Figure 1, reproduced from [8].

\subsection{Silicene}

The difference between silicene (or symmetrically equivalent to it buckled graphene) and graphene for our consideration is due solely to the decreased symmetry of the former. The group of the wavevector at the point $K$ in silicene is $D_{3}$, at the point $\Gamma-D_{3 d}$ (this is also the point group of silicene). The representations of the group $D_{3 d}$ we can obtain on the basis of identity

$$
D_{3 d}=D_{3} \times C_{i}
$$

The direct product has twice as many representations as the group $D_{3}$, half of them being symmetric (denoted by the suffix $g$ ), and the other half antisymmetric (suffix $u$ ) with respect to inversion. The characters of the representations of the group $D_{3 d}$ are presented in the Table 4.

The symmetry analysis in silicene parallels that in graphene, so we'll be brief.

At the point $K$ the functions $\phi_{K}^{A, B}$ realize $E$ graphene, so we'll be brief.

Table 2. Compatibility relations.

\begin{tabular}{ccccccc}
\hline$C_{2} V$ & \multicolumn{3}{c}{$D_{6} h$} & \multicolumn{3}{c}{$D_{3} h$} \\
\hline$A_{1}$ & $A_{1} \mathrm{~g}$ & $B_{2} u$ & $E_{1} u$ & $E_{2} g$ & $A_{1}^{\prime}$ & $E^{\prime}$ \\
$A_{2}$ & $A_{1} \mathrm{u}$ & $B_{2} g$ & $E_{1} g$ & $E_{2} u$ & $A^{\prime \prime}$ & $E^{\prime \prime}$ \\
$B_{1}$ & $B_{1} \mathrm{u}$ & $A_{2} g$ & $E_{1} u$ & $E_{2} g$ & $A_{2}^{\prime}$ & $E^{\prime}$ \\
$B_{2}$ & $A_{2} \mathrm{u}$ & $B_{1} g$ & $E_{1} g$ & $E_{2} u$ & $A^{\prime \prime}$ & $E^{\prime \prime}$ \\
\hline
\end{tabular}

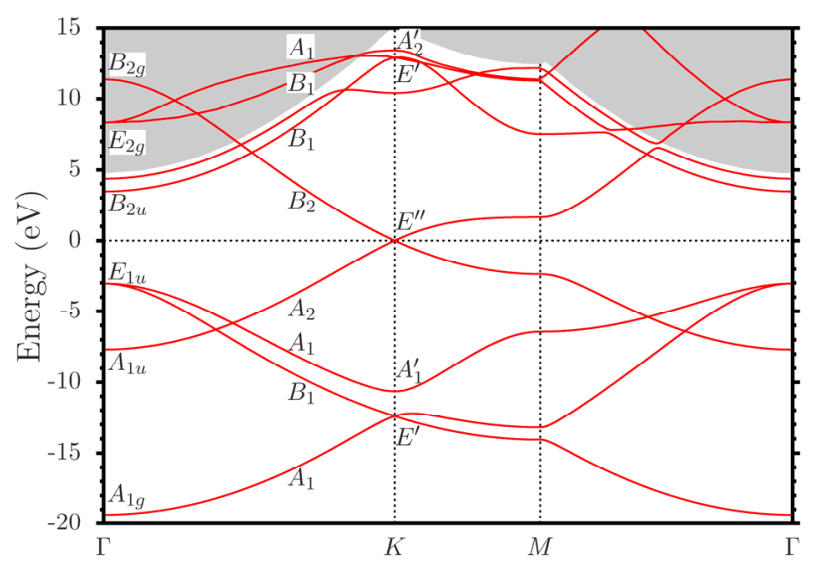

Figure 1. (Color online) Graphene band structure evaluated with use of the FP-LAPW method. The dashed line shows the Fermi energy [reproduced from [8]].
Table 4. Characters table for irreducible representations of $D_{3 d}$ point group.

\begin{tabular}{ccccccc}
\hline Rep & $E$ & $2 C_{3}$ & $3 U_{2}$ & $I$ & $2 S_{6}$ & $3 \sigma_{\mathrm{d}}$ \\
\hline$A_{1 \mathrm{~g}}$ & 1 & 1 & 1 & 1 & 1 & 1 \\
$A_{2 \mathrm{~g}}$ & 1 & 1 & -1 & 1 & 1 & -1 \\
$A_{1 \mathrm{u}}$ & 1 & 1 & 1 & -1 & -1 & -1 \\
$A_{2 \mathrm{u}}$ & 1 & 1 & -1 & -1 & -1 & 1 \\
$E_{\mathrm{g}}$ & 2 & -1 & 0 & 2 & -1 & 0 \\
$E_{\mathrm{u}}$ & 2 & -1 & 0 & -2 & 1 & 0 \\
\hline
\end{tabular}

Orbitals $|s\rangle$ and $|z\rangle$ realize $A_{1}$ representation, and the orbitals $|x, y\rangle$ realize $E$ representation of the group. Thus at the point $K$ the functions $\psi_{s}$ (and $\psi_{z}$ ) realize representation $E$ of the group $D_{3}$. Reducible representation realized by the functions $\psi_{x, y}$ can be decomposed into the irreducible ones:

$$
E \times E=A_{1}+A_{2}+E .
$$

So when the symmetry is reduced by going from graphene to silicene, the representations $A_{1}^{\prime}$ and $A_{2}^{\prime}$ turn into $A_{1}$ and $A_{2}$. Representation $E^{\prime \prime}$ and two representations $E^{\prime}$ turn into three representations $E$. Loosing the reflection in plane symmetry, we can not claim now that one representation is realized exclusively by $|z\rangle$ orbitals. All the $E$ representations mix $|s, p\rangle$ orbitals.

At the point $\Gamma$ the the functions $\phi_{0}^{A, B}$ realizes reducible representation of the group $D_{3 d}$ :

$$
R_{\Gamma}=A_{1 g}+A_{2 u} \text {. }
$$

Orbitals $|s\rangle$ realize $A_{1 u}$, orbitals $|z\rangle-A_{2 u}$, and orbitals $|x, y\rangle-E_{u}$ representations of the group. Thus the functions (1) realize reducible representation of the group $D_{3 d}$ which can be decomposed as

$$
\begin{aligned}
& \left(A_{1 u}+A_{2 u}+E_{u}\right) \times\left(A_{1 g}+A_{2 u}\right) \\
& =A_{1 u}+A_{2 g}+A_{2 u}+A_{1 g}+E_{g}+E_{u} .
\end{aligned}
$$

So when the symmetry is reduced by going from graphene to silicene, the representations $E_{2 g}$ and $E_{1 u}$ turn into the representations $E_{g}$ and $E_{u}$ respectively.

The band structure of silicene is being different from that of graphene, the merging of the bands is no different. The statement becomes clear when comparing Figure 2, reproduced from [10], with Figure 1.

\section{Group Theory Analysis in the Tight-Binding Model with the Spin-Orbit Coupling}

In the absence of spin-orbit coupling, electron spin can be taken into account in a trivial way: each band we considered was doubly spin degenerate.

When the spin-orbit coupling is taken into account, the symmetry, and the representations realized by the sub- 


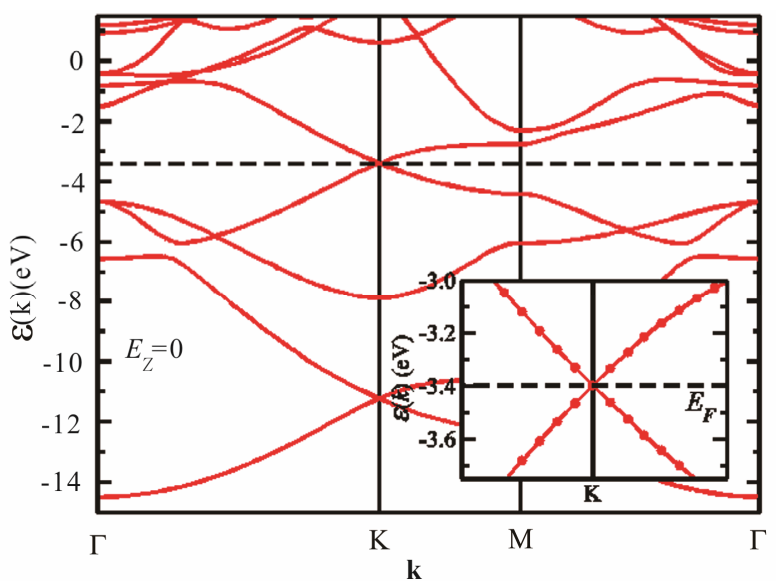

Figure 2. (Color online) DFT-PBE band structures for silicene. The dashed line shows the Fermi energy and the insets show the spectrum near the Fermi level in the vicinity of the $\mathrm{K}$ point [reproduced from [10] with permission].

lattice functions (2) remaim the same. However, instead of atomic orbitals we should consider atomic terms. So for the case of $s p$ hybridization, $\beta$ enumerates states from doublets $|s, p\rangle^{(1 / 2)}$ and quartet $|p\rangle^{(3 / 2)}$.

Due to the semi-integer value of the angular momentum $J$ we have to consider double-valued representations realized by the atomic terms (and by the crystal wave functions). We remind that in this case it is convenient to introduce the concept of a new element of the group (denoted by $Q$ ); this is a rotation through an angle $2 \pi$ about an arbitrary axis, and is not the unit element, but gives the latter when applied twice: $Q^{2}=E$.

The characters of the rotation by angle $\phi$ applied to the term $|\cdot\rangle^{(j)}$ is

$$
\chi^{j}(\phi)=\sin \left(J+\frac{1}{2}\right) \phi / \sin \frac{1}{2} \phi .
$$

With respect to the inversion $I$ the character is

$$
\chi^{j}(I)= \pm(2 J+1),
$$

where the sign plus corresponds to the s states, and the sign minus to the $\mathrm{p}$ states. Finally, the charecters corresponding to reflection in a plane $\sigma$ and rotary reflection through an angle $\phi$ are found writing these symmetry transformations as

$$
\sigma=I C_{2}, \quad S(\phi)=I C(\phi+\pi) .
$$

Both in graphene and in silicene we'll restrict ourselves by the symmetry analysis at the point $K$.

\subsection{Graphene}

The sub-lattice functions $\phi_{K}^{A, B}$ realize $E_{g}$ representation of point group $D_{3 h}$. The electron terms realize two-valued representations of the group, which are presented in Table 5 [17].
Doublet $|s\rangle^{(1 / 2)}$ realizes $\Gamma_{7}$ representation of the group; doublet $|p\rangle^{(1 / 2)}$ realizes $\Gamma_{8}$ representation, quartet $|p\rangle^{(3 / 2)}$ realizes $\Gamma_{7}$ representation twice (We decided to use chemical notation for the single-valued representation, and BSW notation for double-valued representations [9]). The sub-lattice functions realize representation $E_{g}$; from Equation (3) we obtain

$$
\Gamma_{7} \times E_{g}=\Gamma_{8} \times E_{g}=\Gamma_{7}+\Gamma_{8} .
$$

Thus at the point $K$ four bands realize representation $\Gamma_{7}$ of the group $D_{3}^{\prime}$ each, and four bands realize representation $\Gamma_{8}$ each. In particular, we obtained the (well known) result that the four-fold degeneracy (including spin) of the bands merging at the point $K$ is partially removed by the spin-orbit coupling, and only two-fold (Kramers) degeneracy is left.

\subsection{Silicene}

The two-valued representations of $D_{3}^{\prime}$ are presented in Table 6 [17]. Each of the doublets $|s, p\rangle^{(1 / 2)}$ realizes $\Gamma_{4}$ representation of the group. Quartet $|p\rangle^{(3 / 2)}$ realizes this representation twice. The sub-lattice functions realize representation $E$ of the group. From Equation (3) we obtain

$$
\Gamma_{4} \times E=\Gamma_{4}+\Gamma_{5}+\Gamma_{6} .
$$

(For the same reasons as for ordinary representations, two complex conjugate two-valued representations $\Gamma_{5}+\Gamma_{6}$ must be regarded as one physically irreducible representation of twice the dimension).

Thus at the point $K$ four bands which realize representation $\Gamma_{4}$ of the group $D_{3}^{\prime}$ each, and four bands realize representation $\Gamma_{5}+\Gamma_{6}$ each.

\section{Dirac Points}

In this final part of the paper we would like to clarify the relation between the symmetry and the existence of dirac points.

According to the classical approach $[18,19]$, the merging of the bands at a point $\boldsymbol{k}_{0}$ is connected with the multi (higher than one)-dimensional representation of the space group $G_{0}$, realized in this point. Looking for a linear dispersion point in the vicinity of the merging point we may use the degenerate $k \cdot p$ perturbation theory. Let a two-dimensional irreducible representation is realized at a point $\boldsymbol{k}_{0}$. Expanding the wavefunction with respect to the basis of the representation

$$
\psi(k)=\sum_{i=1}^{2} c_{i}(k) \psi_{i}\left(k_{0}\right),
$$

for the expansion coefficients we obtain equation in the form 
Table 3. Characters for two-valued irreducible representations of group $D_{3 h}^{\prime}$.

\begin{tabular}{ccccccccccc}
\hline$D_{3 h}^{\prime}$ & $E$ & $Q$ & $\sigma_{h}$ & $C_{3}$ & $C_{3}^{2}$ & $\mathrm{~S} 3$ & $S_{3}^{2}$ & $3 U_{2}$ & $3 \sigma_{v}$ \\
& & & $\sigma_{h} Q$ & $C_{3} Q$ & $C_{3}^{2} Q$ & $S_{3}^{2} Q$ & $S_{3} Q$ & $3 U_{2} Q$ & $3 \sigma_{v} Q$ \\
\hline$\Gamma_{7}$ & 2 & -2 & 0 & 1 & -1 & $\sqrt{3}$ & $-\sqrt{3}$ & 0 & 0 \\
$\Gamma_{8}$ & 2 & -2 & 0 & 1 & -1 & $-\sqrt{3}$ & $-\sqrt{3}$ & 0 & 0 \\
$\Gamma_{9}$ & 2 & -2 & 0 & -2 & 2 & 0 & 0 & 0 & 0 \\
\hline
\end{tabular}

Table 4. Characters for two-valued irreducible representations of group $D^{\prime}$.

\begin{tabular}{ccccccc}
\hline Rep & $E$ & $Q$ & $C_{3}$ & $C_{3}^{2}$ & $3 U_{2}$ & $3 U_{2} Q$ \\
& & & $C_{3}^{2} Q$ & $C_{3} Q$ & & \\
\hline$\Gamma_{5}$ & 1 & -1 & -1 & 1 & $i$ & $-i$ \\
$\Gamma_{6}$ & 1 & -1 & -1 & 1 & $-i$ & $i$ \\
$\Gamma_{4}$ & 2 & -2 & 1 & -1 & 0 & 0 \\
\hline
\end{tabular}

$$
\sum_{j=1}^{2} \frac{\boldsymbol{k} \cdot \boldsymbol{p}_{i j}}{m} c_{j}(k)=\varepsilon(k) c_{i}(k)
$$

where $p_{i j}=\left\langle\psi_{i}\left(k_{0}\right)|p| \psi_{j}\left(k_{0}\right)\right\rangle$ (of course, we need the absence of inversion symmetry at the point, for the matrix elements to be different from zero). The dispersion law is given by the equation

$$
\varepsilon(\boldsymbol{k})=\sum_{\alpha} a_{\alpha} k_{\alpha} \pm \sqrt{\sum_{\alpha \beta} \gamma_{\alpha \beta} k_{\alpha} k_{\beta}}
$$

where $\alpha, \beta$ are cartesian indexes $x, y$. Equation (24) should contain only combinations of wavevector components which are invariant with respect to all elements of the group $G_{0}$. In the case when the group $G_{0}$ does not have any vector invariants, and the only tensor invariant is the quantity $k_{x}^{2}+k_{y}^{2}$, we obtain the dispersion law

$$
\varepsilon(\boldsymbol{k})= \pm v k,
$$

which, like it was shown by Dirac himself in 1928, guaranties that Equation (25) is Dirac equation, in the sense the the matrices $p_{x}$ and $p_{y}$ satisfy anticommutation relations

$$
p_{x}^{2}=p_{y}^{2} \propto I, \quad\left\{p_{x}, p_{y}\right\}_{+}=0,
$$

where $I$ is the unity matrix.

To be more specific, consider the groups of wavevector at the point $\Gamma$; in graphene it is $D_{3 h}$, and in silicene it is $D_{3}$. In both cases, to find the dispersion law at the point $\Gamma$ it is enough to study invariants of the group $D_{3}$. And we can easily check up that both conditions, necessary for the existence of the Dirac point, are satisfied. This explains, in particular, why the band calcu- lations show the existence of Dirac points in silicene $[9,10]$, which has a lower symmetry than graphene.

In general, the role of the tight binding approximation in symmetry classification of the bands in graphene, like its role in symmetry classification of bands in other crystals, is only auxiliary. The approximation greatly helps in the classification and sheds additional light on the nature of the bands. but one must remember that there are more important things that this or that approximation and this is symmetry.

\section{Conclusion}

This paper presents an applications of group theory to very important cases of graphene and silicene.

\section{Acknowledgements}

Discussions with J. L. Manes, V. Falko and Hua Jiang were very illuminating for the author.

\section{REFERENCES}

[1] K. S. Novoselov, A. K. Geim, S. V. Morozov, D. Jiang, Y. Zhang, S. V. Dubonos, I. V. Grigorieva and A. A. Firsov, "Electric Field Effect in Atomically Thin Carbon Films", Science, Vol. 306, No. 5696, 2004, pp. 666-669. doi:10.1126/science. 1102896

[2] W. M. Lomer, "The Valence Bands in Two-Dimensional Graphite," Proceedings of the Royal Society A, Vol. 227, No. 1170, 1955, pp. 330-349. doi:10.1098/rspa.1955.0014

[3] J. C. Slonczewski and P. R. Weiss, "Band Structure of Graphite," Physical Review, Vol. 109, No. 2, 1958, pp. 272-279. doi:10.1103/PhysRev.109.272

[4] G. Dresselhaus and M. S. Dresselhaus, "Spin-Orbit Interaction in Graphite," Physical Review, Vol. 140, No. 1A, 1965, pp. A401-A412. doi:10.1103/PhysRev.140.A401

[5] F. Bassani and G. P. Parravicini, "Band Structure and Optical Properties of Graphite and of the Layer Compounds GaS and GaSe," Nuovo Cimento B, Vol. 50, No. 1, 1967, pp. 95-128. doi:10.1007/BF02710685

[6] L. M. Malard, M. H. D. Guimaraes, D. L. Mafra, M. S. C. Mazzoni and A. Jorio, "Group-Theory Analysis of Electrons and Phonons in N-Layer Graphene Systems," Physical Review B, Vol. 79, No. 12, 2009, pp. 125426-125433. doi:10.1103/PhysRevB.79.125426

[7] J. L. Manes, "Existence of Bulk Chiral Fermions and 
Crystal Symmetry," Physical Review B, Vol. 85, No. 15, 2012, pp. 155118-115125.

doi:10.1103/PhysRevB.85.155118

[8] E. Kogan and V. U. Nazarov, "Symmetry Classification of Energy Bands in Graphene," Physical Review B, Vol. 85 , No. 11, 2012, pp. 115418-115423. doi:10.1103/PhysRevB.85.115418

[9] C.-C. Liu, H. Jiang and Y. G. Yao, "Low-Energy Effective Hamiltonian Involving Spin-Orbit Coupling in Silicene and Two-Dimensional Germanium and Tin," Physical Review B, Vol. 84, No. 19, 2011, pp. 195430195440. doi:10.1103/PhysRevB.84.195430

[10] N. D. Drummond, V. Zolyomi and V. I. Falko, "Electrically Tunable Band Gap in Silicone," Physical Review B, Vol. 85, 2012, pp. 075423-1-075423-7.

[11] C. Kittel, "Quantum Theory of Solids," John Wiley \& and Sons, Inc., New York, London, 1963.

[12] W. A. Harrison, "Solid State Theory," McGraw Hill Book Company, New York, London, Toronto, 1970.

[13] L. D. Landau and E. M. Lifshitz, "Quantum Mechanics,"
Pergamon Press, Oxford, 1991.

[14] R. S. Knox and S. Gold, "Symmmetry in the Solid State", W. A. Benjamin, Inc., New York, Amsterdam, 1964.

[15] C. Thomsen, S. Reich and J. Maultzsch, "Carbon Nanotubes: Basic Concepts and Physical Properties," Wiley Online Library, Wiley-VCH Verlag GmbH, 2004.

[16] M. I. Petrashen, E. D. Trifonov and J. L. Trifonov, "Applications of Group Theory in Quantum Mechanics," Dover Publications, New York, 2009.

[17] G. F. Koster, J. O. Dimmock, R. G. Wheeler and H. Statz, "Properties of the Thirty-Two Point Groups," MIT Press, Cambridge, 1964.

[18] F. Hund, "Über den Zusammenhang Zwischen der Symmetrie eines Kristallgitters und den Zuständen seiner Elektronen," Zeitschrift für Physik, Vol. 99, No. 1-2, 1936, pp. 119-136. doi:10.1007/BF01847819

[19] C. Herring, "Accidental Degeneracy in the Energy Bands of Crystals," Physical Review B, Vol. 52, No. 4, 1937, pp. 365-373. doi:10.1103/PhysRev.52.365 